\section{(6) OPEN ACCESS}

\title{
Osteoprotegerin reduces the development of pain behaviour and joint pathology in a model of osteoarthritis
}

\author{
Devi Rani Sagar, ${ }^{1}$ Sadaf Ashraf, ${ }^{2}$ Luting $\mathrm{Xu}_{1}{ }^{1}$ James J Burston, ${ }^{1}$ \\ Matthew R Menhinick, ${ }^{3}$ Caroline L Poulter, ${ }^{3}$ Andrew J Bennett, ${ }^{4}$ David A Walsh, \\ Victoria Chapman ${ }^{1}$
}

\begin{abstract}
Handling editor Tore K Kvien
- Additional material is published online only. To view please visit the journal online (http://dx.doi.org/10.1136/ annrheumdis-2013-203260)

${ }^{1}$ Arthritis Research UK Pain Centre, University of Nottingham, Nottingham, UK ${ }^{2}$ School of Medicine, Dentistry and Biomedical Sciences, Queen's University Belfast, Belfast, UK

${ }^{3}$ Faculty of Medicine, University of Nottingham, Nottingham, UK

${ }^{4}$ School of Biomedical Sciences, University of Nottingham, Nottingham, UK
\end{abstract}

\section{Correspondence to} Dr Devi Rani Sagar, Arthritis Research UK Pain Centre, School of Biomedical Sciences, University of Nottingham, Queen's Medical Centre, Nottingham NG7 2UH, UK; devi.sagar@nottingham.ac.uk

Accepted 12 May 2013 Published Online First 30 May 2013

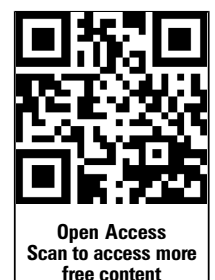
Scan to access m
free content

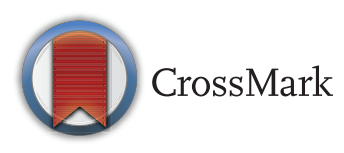

To cite: Sagar DR, Ashraf $S$ Xu L, et al. Ann Rheum Dis 2014;73:1558-1565.

\section{ABSTRACT}

Background Increased subchondral bone turnover may contribute to pain in osteoarthritis (OA).

Objectives To investigate the analgesic potential of a modified version of osteoprotegerin (osteoprotegerin- $\mathrm{Fc}$ $(\mathrm{OPG}-\mathrm{FC}))$ in the monosodium iodoacetate (MIA) model of $O A$ pain.

Methods Male Sprague Dawley rats (140-260 g) were treated with either OPG-Fc (3 mg/kg, subcutaneously) or vehicle (phosphate-buffered saline) between days 1 and 27 (pre-emptive treatment) or days 21 and 27 (therapeutic treatment) after an intra-articular injection of MIA (1 mg/50 $\mu$ l) or saline. A separate cohort of rats received the bisphosphonate zoledronate $(100 \mu \mathrm{g} / \mathrm{kg}$ subcutaneously) between days 1 and 25 post-MIA injection. Incapacitance testing and von Frey (1-15 g) hind paw withdrawal thresholds were used to assess pain behaviour. At the end of the study, rats were killed and the knee joints and spinal cord removed for analysis. Immunohistochemical studies using Iba-1 and GFAP quantified levels of activation of spinal microglia and astrocytes, respectively. Joint sections were stained with haematoxylin and eosin or Safranin-0 fast green and scored for matrix proteoglycan and overall joint morphology. The numbers of tartrate-resistant acid phosphatase-positive osteoclasts were quantified. $\mathrm{N}=10$ rats/group.

Results Pre-emptive treatment with OPG-Fc significantly attenuated the development of MIA-induced changes in weightbearing, but not allodynia. OPG-Fc decreased osteoclast number, inhibited the formation of osteophytes and improved structural pathology within the joint similarly to the decrease seen after pretreatment with the bisphosphonate, zoledronate. Therapeutic treatment with OPG-Fc decreased pain behaviour, but did not improve pathology in rats with established joint damage.

Conclusions Our data suggest that early targeting of osteoclasts may reduce pain associated with OA.

\section{INTRODUCTION}

Pain is one of the first symptoms of knee osteoarthritis $(\mathrm{OA}),{ }^{1}$ and often causes increasing disability as the disease progresses. Treatments for OA pain have limited efficacy, and joint replacement surgery is a common outcome. ${ }^{2} 3$ OA pain arises both from peripheral nociceptive inputs, and from central sensitisation. ${ }^{45}$ Central sensitisation usually decreases after total joint replacement surgery, ${ }^{2}$ indicating that continuing nociceptive inputs from the joint may drive central pain mechanisms. Within the joint, OA pain is associated with subchondral bone marrow lesions ${ }^{6}$ and with synovitis (see references in Sellam and Berenbaum ${ }^{7}$ ). Several existing treatments may partially alleviate OA pain by reducing synovitis, but only recently has the analgesic potential of targeting subchondral bone attracted attention.

$\mathrm{OA}$ is associated with an initial increase in subchondral bone resorption and resultant thinning of the subchondral plate, followed by subchondral sclerosis and osteophyte formation. ${ }^{8}$ Changes in subchondral bone turnover precede changes in cartilage (see references in Kwan Tat $e t a l^{9}$ ) and painrelated molecules have been detected in OA subchondral bone. ${ }^{10}$ Dynamic changes in bone turnover result from increased activity of osteoclasts and osteoblasts, and the osteoprotegerin (OPG)/receptor activator of NF-kB (RANK)/receptor activator of $\mathrm{NF}-\kappa \mathrm{B}$ ligand (RANKL) system is critical for this activity. ${ }^{9} 11 \quad 12$ Osteoclast activation is associated with reduced extracellular $\mathrm{pH}$, and release of proteases. In addition to increasing bone resorption, osteoclast products can activate and sensitise peripheral nerves and increase pain signalling. ${ }^{13}$ Furthermore, subchondral osteoclasts cut channels through the osteochondral junction (OCJ), and this loss of osteochondral integrity may expose subchondral nerves to algesic factors produced by the synovium and articular cartilage. ${ }^{14}$ Bisphosphonates, which inhibit bone resorption, increase trabecular bone thickness, and may attenuate bone marrow lesions and knee pain, ${ }^{15}$ supporting the hypothesis that increased subchondral bone turnover may contribute to OA pain. The beneficial effects of strontium ranelate on structural changes and pain in patients with OA, further support a contribution of bone turnover to OA progression. ${ }^{16}$

As in human OA, both surgical ${ }^{17}$ and the monosodium iodoacetate (MIA) ${ }^{18-20}$ models of OA in the rodent exhibit excessive osteoclast activity and bone remodelling. Chronic bisphosphonate treatment inhibits osteoclast number, bone resorption and structural changes ${ }^{17} 20$ in these models and a canine model ${ }^{21}$ of OA. Importantly, chronic preemptive treatment with zoledronate attenuated both the pathological features of $\mathrm{OA}$ and the 
development of pain behaviour. ${ }^{20}$ A recent clinical trial indicated reduced OA knee pain after treatment with strontium ranelate, an effect that may be mediated through reduced bone turnover. ${ }^{16}$ The analgesic effects of bisphosphonates or strontium in patients with OA, however, are relatively weak, ${ }^{15} 1622$ and more potent or specific inhibitors of subchondral bone turnover may have greater analgesic potential in OA.

OPG is a soluble member of the tumour necrosis factor superfamily which sequesters RANKL and puts a brake on osteoclast formation (see references in Kwan Tat et $a l^{9}$ ). An imbalance in the OPG/RANKL system, both in synovial fluid and serum, has been associated with OA severity in man. ${ }^{12} 23$ Exogenous treatment with a modified OPG (OPG-Fc) attenuates bone loss in a rodent model of inflammatory arthritis ${ }^{24}$ and, although effects on arthritis pain were not reported, OPG inhibited bone cancerinduced pain behaviour. ${ }^{25}$ We hypothesised that OPG-Fc could prevent the development of pain behaviour in a model of OA pain by inhibiting osteoclastogenesis and reducing early bone turnover in the joint. We therefore compared the effects of preemptive and therapeutic OPG on pain and joint pathology in a model of OA.

\section{METHODS}

Studies were conducted in accordance with UK Home Office Animals (Scientific Procedures) Act (1986) and guidelines of the International Association for the Study of Pain. One hundred and four male Sprague Dawley rats (Charles River UK) weighing $140-260 \mathrm{~g}$ (5-7 weeks old) were used. An overview of the experimental design and the group sizes is provided in online supplementary figure S1 and online supplementary table S1. Rats were briefly anaesthetised with isoflurane $\left(3 \%\right.$ in $\left.1 \mathrm{l} / \mathrm{min} \mathrm{O}_{2}\right)$. Joint damage was induced by a single intra-articular injection of MIA ( $1 \mathrm{mg} / 50 \mu \mathrm{l}$; Sigma UK) in saline through the infrapatellar ligament of the left knee. OA develops in response to a variety of insults to the joint. Intra-articular MIA disrupts glycolysis resulting in chondrocyte death, ${ }^{26}$ which is believed to be the primary insult in our model. Control animals received a single injection of saline $(50 \mu \mathrm{l})$ into the left knee. Two separate measures of pain behaviour were assessed: change in hind limb weight distribution, which is a behavioural correlate of hyperalgesia, and hind paw mechanical withdrawal thresholds, which provide an index of central sensitisation. Changes in hindlimb weight distribution and hindpaw mechanical withdrawal thresholds were measured before intra-articular injection (day 0, baseline) and then from day 3 onwards. ${ }^{27}$ Weight distribution was assessed using an incapacitance tester (Linton Instrumentation, UK) and paw withdrawal thresholds (PWTs) were assessed using von Frey monofilaments (Semmes-Weinstein monofilaments of bending forces $1-15 \mathrm{~g}) .{ }^{28}$ Under isoflurane anaesthesia $(1.5-2 \%$ in $66 \%$ $\mathrm{N}_{2} \mathrm{O}-33 \% \mathrm{O}_{2}$ ), neuropathic rats received tight ligation of spinal nerves L5 and L6 with 6-0 silk thread, as previously described. ${ }^{29}$ Sham rats had nerves exposed but not ligated. PWTs were assessed as above, before surgery and then from day 2 to 14 .

\section{Drug administration}

A modified version of OPG (consisting of the RANKL-binding portion of OPG linked with the constant $(\mathrm{Fc})$ domain of $\operatorname{IgG}^{24}$ ), OPG-Fc, was a gift from Amgen Ltd and was diluted to $3 \mathrm{mg} / \mathrm{ml}$. Zoledronate tetrahydrate (Toronto Research Chemicals) was made up as a $100 \mu \mathrm{g} / \mathrm{ml}$ solution. Rats were randomised in the treatment protocol, with the tester blind to both drug and injury. Dosing regimens were based on pharmacokinetics and previous evidence of efficacy in rodent models for each of OPG- $\mathrm{Fc}^{24}$ and zoledronate. $^{20}$

\section{Pre-emptive OPG-Fc}

Subcutaneous injections of OPG-Fc $(3 \mathrm{mg} / \mathrm{kg})$ or vehicle (phosphate-buffered saline (PBS)) were given every other day from days 1 to 27 post-MIA injection. Saline-treated rats received vehicle.

\section{Pre-emptive zoledronate}

Subcutaneous injections of zoledronate $(100 \mu \mathrm{g} / \mathrm{kg})$ or vehicle (PBS) were given every third day from days 1 to 25 post-MIA injection, according to Strassle et al. $^{20}$ Saline-treated rats received vehicle.

\section{Therapeutic OPG-Fc}

Subcutaneous injections of vehicle were given every other day from days 1 to 20 post-MIA injection. On day 21 rats received either OPG-Fc (3 mg/kg) or vehicle (PBS) every other day until day 27. Rats were stratified according to their pain behaviour before drug treatment (days 14 and 16).

\section{Histology}

At the end of the pharmacological study, rats were killed by anaesthetic overdose and tissues were fixed by transcardiac perfusion with $4 \%$ paraformaldehyde solution. Tibiofemoral joints were removed and post-fixed in neutral buffered formalin (containing 4\% formaldehyde), decalcified in EDTA and processed as previously described. ${ }^{30}$ Mid-coronal tissue sections $(5 \mu \mathrm{M})$ were stained for evaluations and all histomorphometry was by an observer blinded to the treatment groups. Lumbar spinal cords were post-fixed in paraformaldehyde, as previously described. $^{28}$

\section{Joint structure and inflammation}

Haematoxylin and eosin or Safranin-O/fast green-stained sections were scored for matrix proteoglycan and overall joint morphology. ${ }^{31} 32$ Cartilage surface integrity was scored from 0 (normal) to 5 (full-thickness degeneration), and a total joint damage score (range 0-15) was calculated as cartilage surface integrity $\times$ length of cartilage involved in thirds. Osteophyte scores ranged from 0 (no osteophyte) to 3 (osteophyte $>160 \mu \mathrm{m})$. The integrity of the OCJ was measured as the number of channels crossing the OCJ into the cartilage of the whole section of medial tibial plateau. Proteoglycan loss was evaluated on a scale from 0 (normal proteoglycan staining) to 4 (complete loss of proteoglycan staining). Chondrocyte appearance was graded from 0 (normal appearance and density), to 3 (chondrocyte hypocellularity). Synovial inflammation was graded as previously described ${ }^{33}$ on a scale from 0 (lining cell layer $1-2$ cells thick) to 3 (lining cell layer $>9$ cells thick and/or severe increase in cellularity).

\section{Osteoclast numbers}

Tissue sections were dewaxed and recalcified before tartrate-resistant acid phosphatase (TRAP) staining using the commercially available kit (Sigma-Aldrich, UK). The number of TRAP-positive multinucleated osteoclasts was quantified within the subchondral bone area comprising the area between the cartilage/bone junction and the growth plate following the standard convention. $^{34}$

\section{Quantification of glial activation in the spinal cord}

Microglial cells were stained using Iba- $1^{35}$ and astrocytes were stained using glial fibrillary acidic protein (GFAP), ${ }^{36}$ as previously described. ${ }^{28}$ Total numbers of positively identified 
activated microglia expressing Iba- 1 were counted manually in both ipsilateral and contralateral quadrants of individual $40 \mu \mathrm{m}$ sections. For quantification of GFAP, images of the superficial dorsal horn of the spinal cord were captured using a $\times 40$ objective lens. All image analysis, cell counts and fluorescence measurements were performed as previously described. ${ }^{28}$

\section{Statistical analyses}

For all comparisons, $\mathrm{p}<0.05$ was taken to indicate statistical significance. Statistical comparisons between groups were carried out using a two-way analysis of variance or Kruskal-Wallis tests with post hoc $t$ tests, Dunnett's or Mann-Whitney tests, as appropriate. Bonferroni corrections were used for multiple comparisons. Data are presented graphically as means $( \pm$ SEM).

\section{RESULTS}

\section{Pre-emptive treatment with OPG-Fc reduced pain behaviour and pathology in osteoarthritic rats}

Consistent with previous studies, MIA-treated rats which received vehicle exhibited a decrease in ipsilateral weightbearing and hind PWTs from day 3 post-MIA injection, which was maintained throughout the study and was significant compared with saline-injected rats (figure 1A,B). At 28 days post-MIA injection, there was an increase in the number of TRAP-positive osteoclasts in the tibial plateau (figure 1C), increased synovial inflammation, cartilage damage and osteophyte formation, compared with saline-injected rats (figures 1D-F and 2). Intra-articular injection of saline was not associated with pain behaviour (figure 1A,B), nor changes in joint structure (figures 1D-F and 2). Pre-emptive systemic OPG-Fc significantly attenuated the development of MIA-induced changes in weightbearing from day 16 onwards, compared with vehicle administration in MIA-treated rats (figure 1A). Pre-emptive OPG-Fc did not significantly alter MIA-induced decreases in hind PWTs (figure 1B). Pre-emptive systemic OPG-Fc significantly decreased the number of TRAP-positive osteoclasts (see online supplementary figure S2) in the tibial plateau, synovitis, cartilage damage and osteophyte formation in MIA-treated rats, compared with vehicle treatment (figures $1 \mathrm{C}-\mathrm{F}$ and 2). However, neither pre-emptive nor therapeutic treatment with OPG-Fc significantly altered the MIA-induced increase in the number of channels crossing the OCJ, nor did these treatments alter the extent of MIA-induced loss of proteoglycan or the chondrocyte appearance (table 1, figure 2, see online supplementary figure S3).

In contrast to the marked effects of pre-emptive OPG-Fc treatment on MIA-induced changes in weightbearing, synovitis, osteophytosis and aspects of cartilage damage, there was no evidence that this treatment alters spinal mechanisms of central sensitisation. At 28 days post-MIA injection, there was a significant increase in the number of Iba-1-positive microglia and the level of GFAP immunofluorescence, indicative of reactive astrocytes, in the ipsilateral dorsal horn of MIA-treated rats which received pre-emptive vehicle $(36.92 \pm 3.5$ Iba-1-positive microglia, GFAP immunofluorescence: $36.56 \pm 0.7$ mean grey intensity value AU) compared with saline-injected controls $(21.46 \pm 1.8$ Iba-1-positive microglia, GFAP immunofluorescence: $24.57 \pm 2.5$ mean grey intensity value AU). Pre-emptive systemic OPG-Fc did not significantly alter the expression of these markers of central sensitisation (31.63 \pm 1.9 Iba-1-positive microglia, GFAP immunofluorescence: $35.45 \pm 1.0$ mean grey intensity value AU) in MIA-injected rats. To consolidate the evidence that the effects of pre-emptive treatment with OPG-Fc were specific to joint pain, rather than a generalised anti-nociceptive effect, the effect of OPG-Fc in another model of chronic pain was evaluated.
Pre-emptive treatment with OPG-Fc did not significantly alter the decrease in hind PWTs in neuropathic rats (see online supplementary figure S4).

\section{Pre-emptive treatment with zoledronate reduced pain behaviour and pathology in osteoarthritic rats}

In the next series of experiments we sought to further strengthen the association between subchondral bone turnover and changes in weightbearing in this model of OA. Pre-emptive systemic zoledronate significantly attenuated the MIA-induced changes in weightbearing compared with vehicle-treated MIA rats (figure $3 \mathrm{~A})$. Zoledronate produced an elevation in hind PWTs in MIA-treated rats compared with vehicle-treated MIA rats, but this did not reach significance (figure $3 \mathrm{~A}$ ). There was no effect of zoledronate on the number of TRAP-positive osteoclasts in the tibial plateau in MIA-treated rats (figure 3A). Zoledronate significantly inhibited the development of synovitis, cartilage pathology and osteophyte formation compared with vehicle administration in MIA-treated rats (figure 3D-F). Zoledronate significantly decreased the MIA-induced increase in the number of channels crossing the OCJ (table 1), but, consistent with OPG-Fc, zoledronate did not alter the MIA-induced proteoglycan loss or the chondrocyte appearance (table 1).

\section{Therapeutic treatment with OPG-Fc}

Finally, the therapeutic effects of OPG-Fc once the model of OA pain is established were determined. Before intervention, MIA-treated rats developed changes in weightbearing and hind PWTs at similar rates and magnitudes as the earlier experimental groups (figure 4A,B). Therapeutic OPG-Fc from day 21 to 27 significantly inhibited any further decreases in weightbearing on the ipsilateral hindlimb, compared with vehicle treatment, but did not reverse the decrease in weightbearing exhibited before OPG-Fc intervention (figure 4A). Therapeutic OPG-Fc did not alter changes in hind PWTs in MIA-treated rats (figure 4B). Therapeutic OPG-Fc significantly reduced the number of osteoclasts in the ipsilateral knee joint, compared with vehicle-treated MIA rats, but did not significantly alter the extent of synovial inflammation, cartilage pathology or osteophyte number at day 28 (figure 4C-F, see online supplementary figure S3).

\section{DISCUSSION}

Here we demonstrate for the first time that a modified form of the endogenous inhibitor of RANKL, OPG-Fc, which attenuates osteoclastogenesis, decreases the number of subchondral osteoclasts and inhibits the development of pain behaviour (weightbearing asymmetry) and aspects of joint pathology in the MIA model of OA pain. OPG-Fc treatment did not alter MIA-induced changes in hind PWTs (distal allodynia). Therapeutic treatment with OPG-Fc, over a relatively short period of time, prevented further changes in weightbearing asymmetry and attenuated osteoclast number but not other structural changes within the joint. Differences in the effects of pre-emptive versus therapeutic OPG-Fc on weightbearing asymmetry and structural changes within the joint suggest that this treatment may preferentially ameliorate early structural progression and pain associated with OA.

Pain is the main clinical symptom of OA, and may result from both increased nociceptive input from the joint, and sensitisation within the spinal cord and brain. Pathology within a number of joint structures, including the highly innervated subchondral bone and synovium ${ }^{14}$ is likely to drive nociceptive inputs associated with OA. Our findings that OPG-Fc reduced the number of subchondral osteoclasts, chondropathy and 
A

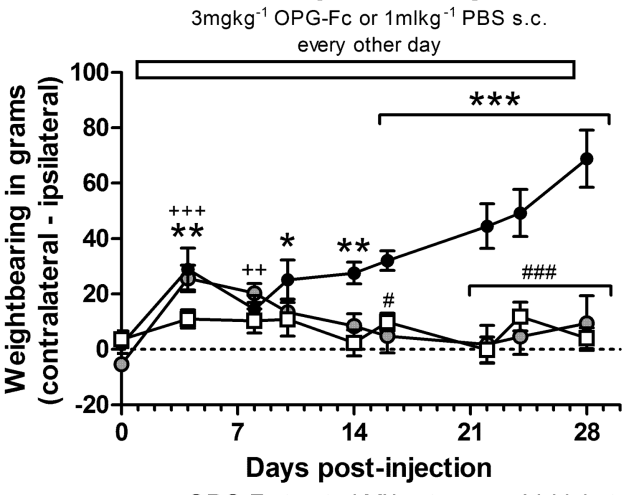

- OPG-Fc treated MIA rats

C

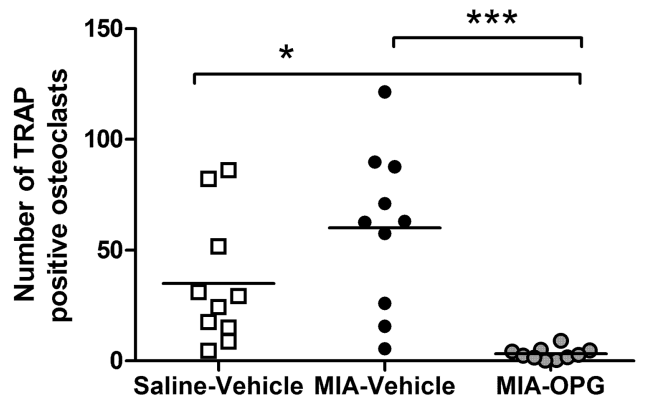

E

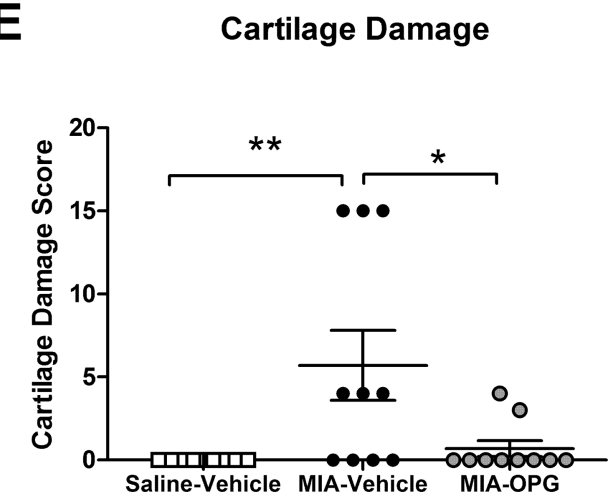

B Hind paw Withdrawal Threshold

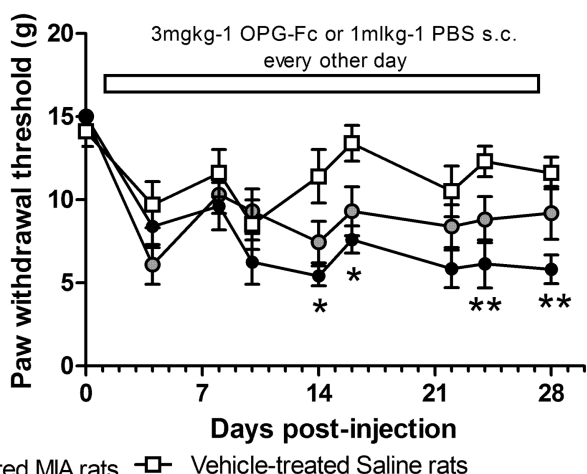

D Synovial Inflammation

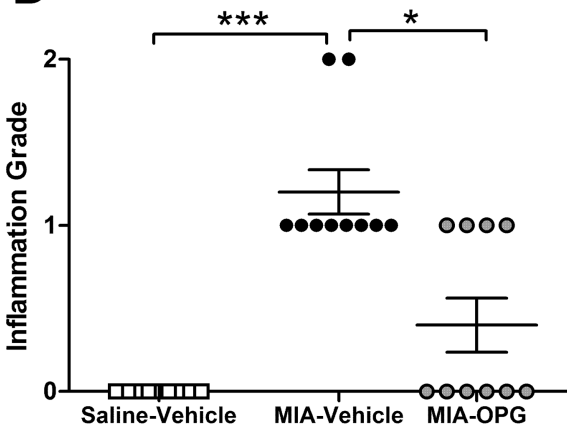

F Osteophyte Number

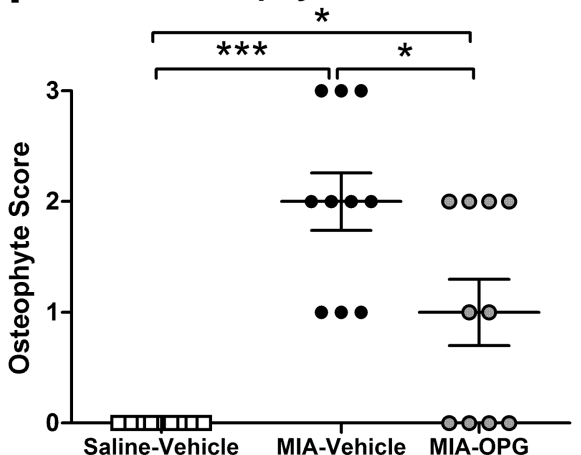

Figure 1 Pre-emptive treatment with osteoprotegerin (OPG)-Fc significantly attenuates monosodium iodoacetate (MIA)-induced changes in (A) weightbearing, but not (B) mechanical allodynia and is accompanied by a significant reduction in (C) osteoclast number, (D) synovial inflammation (E) cartilage damage and (F) osteophyte number. Statistical comparison between groups was carried out using a two-way analysis of variance (A) or Kruskal-Wallis test (B) ${ }^{*} \mathrm{p}<0.05,{ }^{* *} \mathrm{p}<0.01,{ }^{* * *} \mathrm{p}<0.001$ for MIA-vehicle versus saline-vehicle, ${ }^{++} \mathrm{p}<0.01,{ }^{+++} \mathrm{p}<0.001$ for MIA-OPG versus saline-vehicle, ${ }^{\#} \mathrm{p}<0.05,{ }^{\# \#} \mathrm{p}<0.001$ for MIA-OPG versus MIA-vehicle. (C-F) Statistical comparison comparing groups were performed using a Kruskal-Wallis test ${ }^{*} p<0.05, * * * p<0.001$. All data are presented as mean $\pm S E M, n=10$ rats per group. For osteoclast numbers six sections for each rat were quantified and data are presented as mean \pm SEM. PBS, phosphate-buffered saline; TRAP, tartrate-resistant acid phosphatase.

osteophyte formation in the MIA model of OA lead us to suggest that effects of OPG-Fc on pain behaviour may be mediated by peripheral effects within the joint. As discussed earlier, the primary insult in the MIA model is chondrocyte death, and further work would be required to determine whether our findings can be generalised to OA induced by other factors.

Distal allodynia (decreased hindpaw mechanical withdrawal thresholds) and the activation of spinal neuroimmune cells are each indicative of altered spinal processing of sensory inputs and central sensitisation in MIA-treated rats. ${ }^{28}$ These changes parallel evidence of abnormal central pain processing in human OA. ${ }^{5}$ OPG-Fc did not reduce numbers of activated neuroimmune cells in the spinal cord in the MIA model of OA pain, and did not significantly decrease distal allodynia. By contrast, pre-emptive and therapeutic treatment with a non-steroidal anti-inflammatory drug reduced weightbearing asymmetry, distal allodynia and spinal gliosis in this model of OA pain, ${ }^{28}$ demonstrating the importance of a peripheral and central site of action, in particular once OA pain and structural changes to the joint are established.

Pre-emptive OPG-Fc treatment did not significantly alter mechanical allodynia either in the model of OA or in a model of neuropathic pain, further suggesting that the effects of OPG-Fc are predominantly mediated by a peripheral site of action. This is in contrast to the inhibitory effects of OPG on neuroimmune cell activation in the spinal cord in a model of bone cancer pain. ${ }^{25}$ Differences in the effects of OPG on spinal sensitisation mechanisms possibly reflect distinct pain 

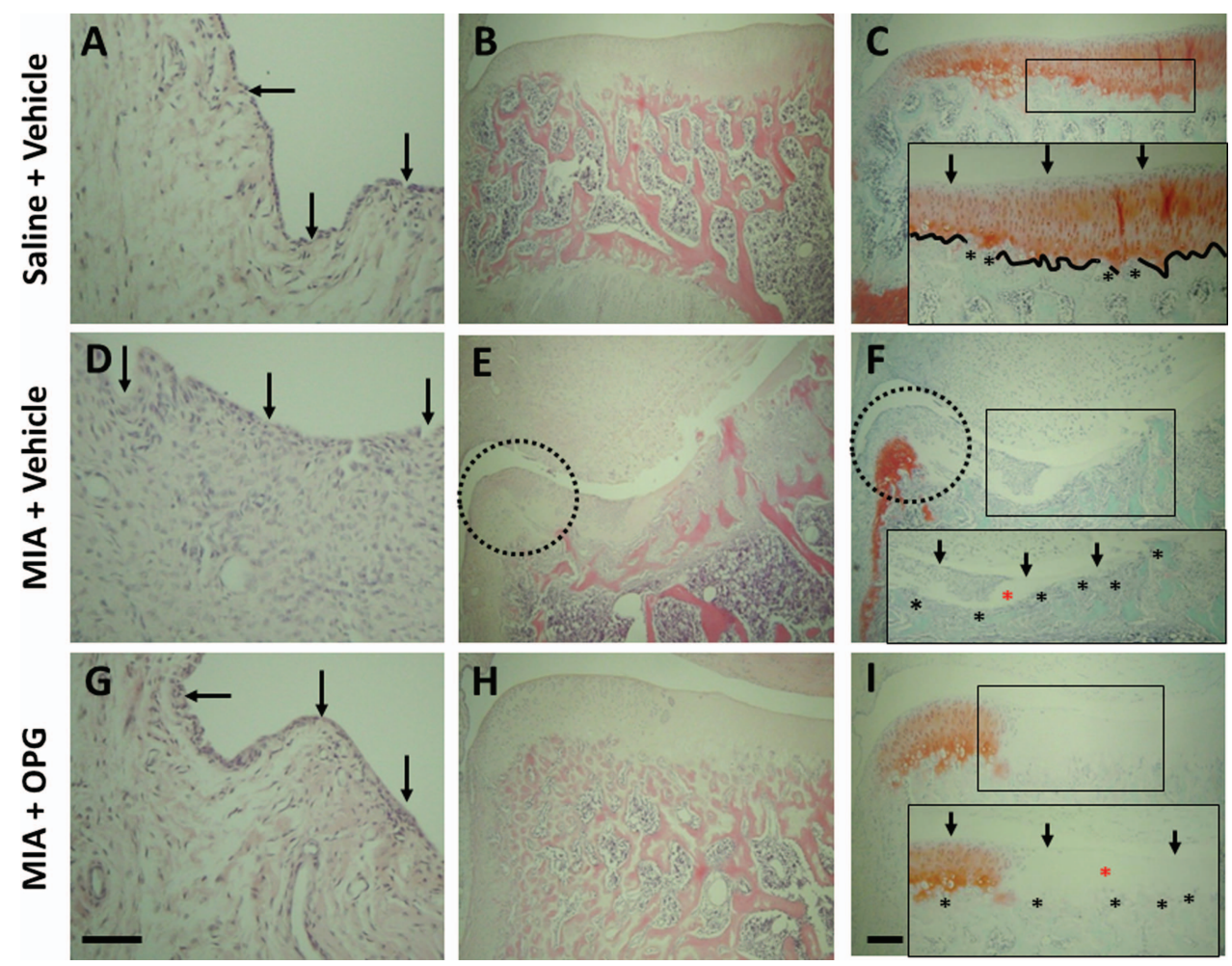

Figure 2 Pre-emptive osteoprotegerin (OPG)-Fc attenuates the development of histopathological changes in the knee joints of monosodium iodoacetate (MIA)-treated rats. Non-arthritic saline injected control rats $(A-C)$ showed normal synovia, with 1-2 cell thick synovial lining (A; arrows), normal smooth cartilage and joint margins (B-C; arrows) with normal chondrocyte distribution and proteoglycan staining (C). Fewer channels (asterisks) crossing the osteochondral junction (black line) were seen in these animals (C). MIA-injected rats (D-F) had thicker synovial lining (arrows) and increased cellularity throughout the synovia (D). Severe cartilage loss and osteophyte growth (circle) at joint margin (E and F) accompanied with chondrocyte hypocellularity and severe loss of proteoglycan staining as well as increased number of channels (asterisks) crossing the osteochondral junction were seen in these animals (F). OPG-Fc treated arthritic rats (G-I) had normal synovial cellularity (G; arrows) with smooth articular cartilage ( $\mathrm{l}$; arrows) and less pronounced osteophytes at joint margins (H). Nevertheless, chondrocyte hypocellularity, proteoglycan loss and channels entering the cartilage in $\mathrm{OPG}-\mathrm{Fc}$-treated arthritic rats (I) were comparable to MIA-injected rats (F). Coronal sections stained with haematoxylin and eosin (synovia: A, D, G, joint: B, E, H) and Safranin- $0(C, F, I)$ stains. Bars $=100 \mu \mathrm{m}$.

mechanisms associated with these two types of bone pathologies. Clinical benefits of joint replacement surgery indicate that peripheral mechanisms are particularly important in OA pain, highlighting the potential for relieving OA pain by treatments directed at the joint itself.

The attenuation of MIA-induced synovitis by pre-emptive OPG-Fc is consistent with osteoclasts modifying the biochemical environment of peripheral nerve terminals within the subchondral bone. Indeed, the active resorption of bone by osteoclasts is achieved by maintaining an acidic extracellular environment at the osteoclast/bone interface, which probably activates acid sensing channels, and sensitises other pro-nociceptive/inflammatory cation channels such as TRPV1 expressed by primary afferent nociceptors innervating the subchondral bone (see references in Jimenez-Andrade et $a l^{13}$; Nagae et $a l^{37}$ ). Given that synovitis is associated with OA pain clinically, the reduction in MIA-induced synovitis by pre-emptive OPG-Fc may contribute to its effects on pain behaviour in our study. Once the model was established, a relatively short (7 days) treatment with OPG-Fc did not alter synovial inflammation, suggesting that the

Table 1 Effects of OPG-Fc or zoledronate on the number of channels in the osteochondral junction (OCJ) in the medial tibial plateaus (MTPs), chondrocyte appearance and proteoglycan loss in MIA-treated rats compared with MIA-vehicle-treated and saline-treated rats.

\begin{tabular}{|c|c|c|c|c|c|c|c|c|}
\hline & \multicolumn{3}{|c|}{ Pre-emptive OPG } & \multicolumn{2}{|c|}{ Therapeutic OPG } & \multicolumn{3}{|c|}{ Pre-emptive zoledronate } \\
\hline & Saline-vehicle & MIA-vehicle & MIA-OPG & MIA-vehicle & MIA-OPG & Saline-vehicle & MIA-vehicle & MIA-zoledronate \\
\hline $\begin{array}{l}\text { Number of channels crossing the } \mathrm{OCJ} \text { in } \\
\text { MTP }\end{array}$ & $4 \pm 0.4$ & $8 \pm 1$ & $7 \pm 2$ & $7 \pm 1$ & $6 \pm 0.4$ & $1 \pm 0.3$ & $7 \pm 1^{* * *}$ & $3 \pm 0.6^{*+}$ \\
\hline Chondrocyte appearance score & $0.3 \pm 0.2$ & $2 \pm 0.3^{* *}$ & $3 \pm 0.2^{* * *}$ & $3 \pm 0.3$ & $2 \pm 0.3$ & $0 \pm 0$ & $3 \pm 0^{* * *}$ & $3 \pm 0.2^{* * *}$ \\
\hline Proteoglycan loss grade & $1.2 \pm 0.3$ & $3 \pm 0.4^{*}$ & $3 \pm 0.3^{*}$ & $4 \pm 0$ & $4 \pm 0$ & $0.3 \pm 0.2$ & $4 \pm 0.2^{* * *}$ & $3 \pm 0.2^{* * *}$ \\
\hline
\end{tabular}

Statistical analysis was performed with a Kruskal-Wallis test.

${ }^{*} p<0.05,{ }^{* *} p<0.01,{ }^{* *} p<0.001$ versus saline-vehicle; ${ }^{+} p<0.05$ versus MIA-vehicle.

Data are expressed as mean \pm SEM per section.

MIA, monosodium iodoacetate; OPG, osteoprotegerin. 


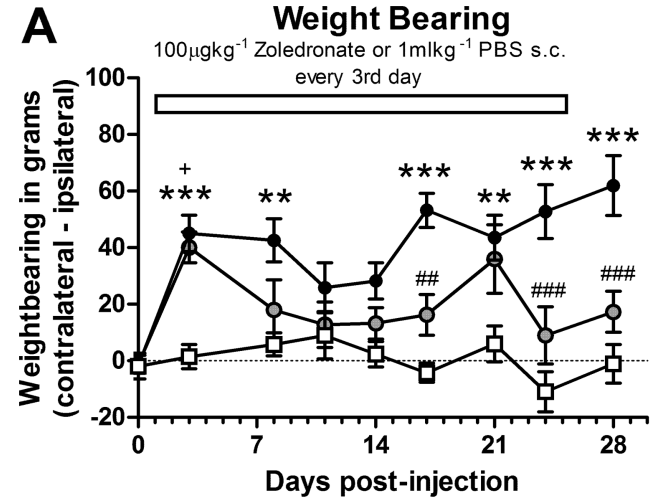

Days post-injection
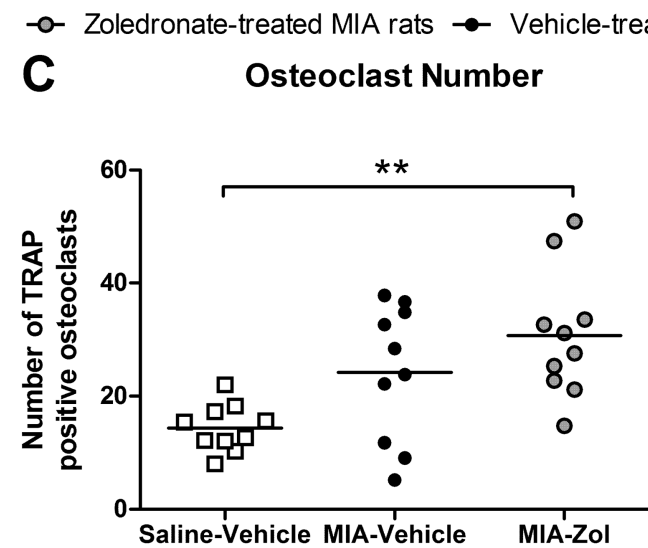

E

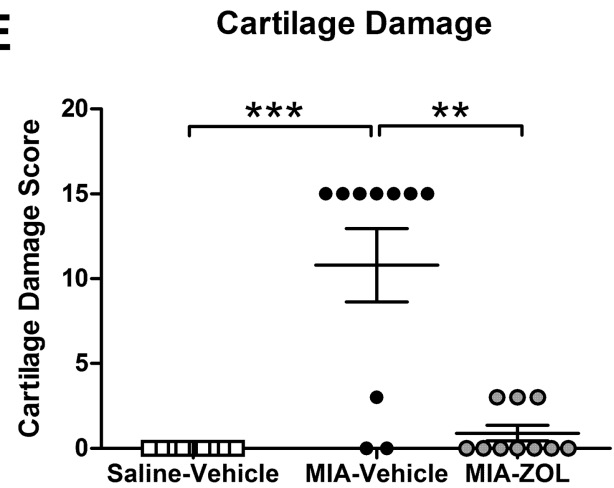

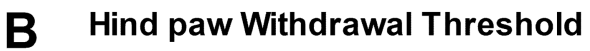

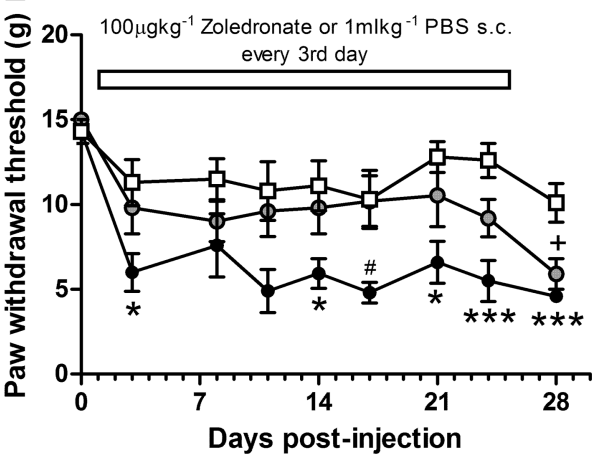

$\rightarrow-$ Vehicle-treated Saline rats

D Synovial Inflammation
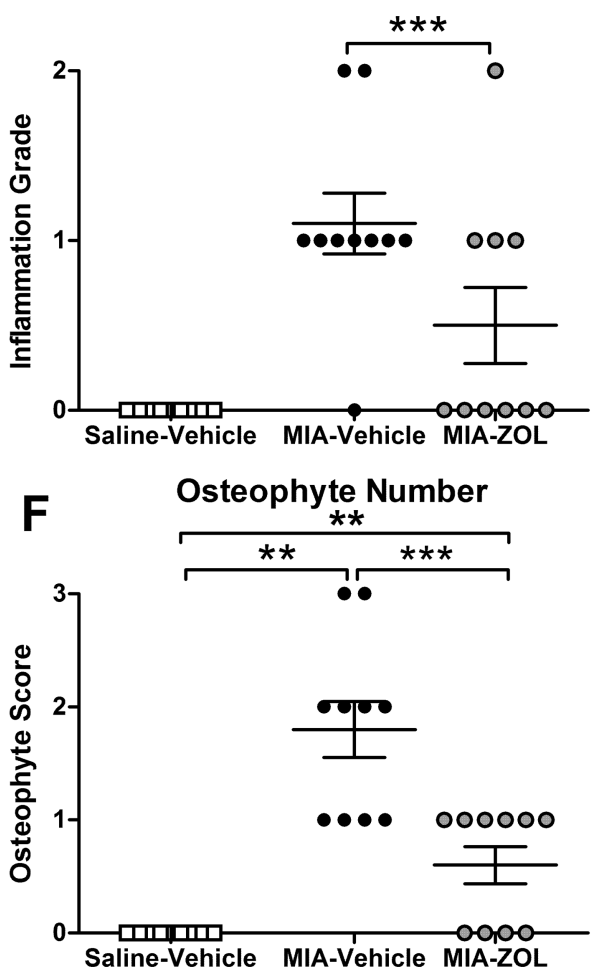

Figure 3 Pre-emptive treatment with zoledronate (Zol) significantly attenuates monosodium iodoacetate (MIA)-induced changes in (A) weightbearing, but not (B) mechanical allodynia with no change in (C) osteoclast number, but significant reductions in (D) synovial inflammation (E) cartilage damage and (F) osteophyte number. (A and B) Statistical comparison between groups was carried out using a two-way analysis of variance $(A)$ or Kruskal-Wallis test $(B)$ where ${ }^{*} p<0.05,{ }^{* *} p<0.01,{ }^{* * *} p<0.001$ for MIA-vehicle versus saline-vehicle, ${ }^{+} p<0.05$ for MIA-zoledronate versus saline-vehicle, ${ }^{\#} p<0.05,{ }^{\# \#} p<0.01,{ }^{\# \# \#} p<0.001$ for MIA-zoledronate versus MIA-vehicle. (C-F) Statistical comparison comparing groups were performed using a Kruskal-Wallis test ${ }^{*} p<0.05,{ }^{* *} p<0.01,{ }^{* *} p<0.001$. All data are presented as mean $\pm S E M, n=10$ rats per group. For osteoclast numbers six sections for each rat were quantified and data are presented as mean \pm SEM. PBS, phosphate-buffered saline.

effects of pre-emptive OPG-Fc on synovitis may be indirectfor example, via an attenuation of subchondral bone turnover or cartilage pathology.

The inhibitory effects of pre-emptive treatment with the bisphosphonate zoledronate reported herein are broadly comparable to those previously published, ${ }^{20}$ with reduced OA structural damage and pain behaviour evident. Furthermore, as with OPG-Fc, zoledronate pretreatment significantly attenuated weightbearing asymmetry, but less so distal allodynia. The precise mechanisms by which structural pathology may mediate OA pain remain uncertain, both in man and in animal models. Pain progression in OA is accompanied by an early bone loss, followed by a later sclerosis of the subchondral bone plate (see references in Kwan Tat et $a l^{9}$ ). Increased subchondral uptake of radiolabelled bisphosphonates ${ }^{38}$ and MRI-defined bone marrow lesions ${ }^{6}$ are each associated with $\mathrm{OA}$ pain, and subchondral osteoclast numbers are increased in OA both in $\operatorname{man}^{10}$ and rats. $^{2039}$ Subchondral bone pathology may contribute to cartilage damage in OA (see references in Zhang et $a l^{40}$ ). Furthermore, osteophytes are innervated by sensory nerves originating in the subchondral bone, and may be a source of OA pain. ${ }^{41}$ Our data suggest that effects on structural factors such as synovitis, cartilage damage and osteophytosis may contribute to the inhibitory effects of OPG-Fc and zoledronate on pain behaviour in our model. However, reductions in pain behaviour occurred despite persistently abnormal proteoglycan staining 

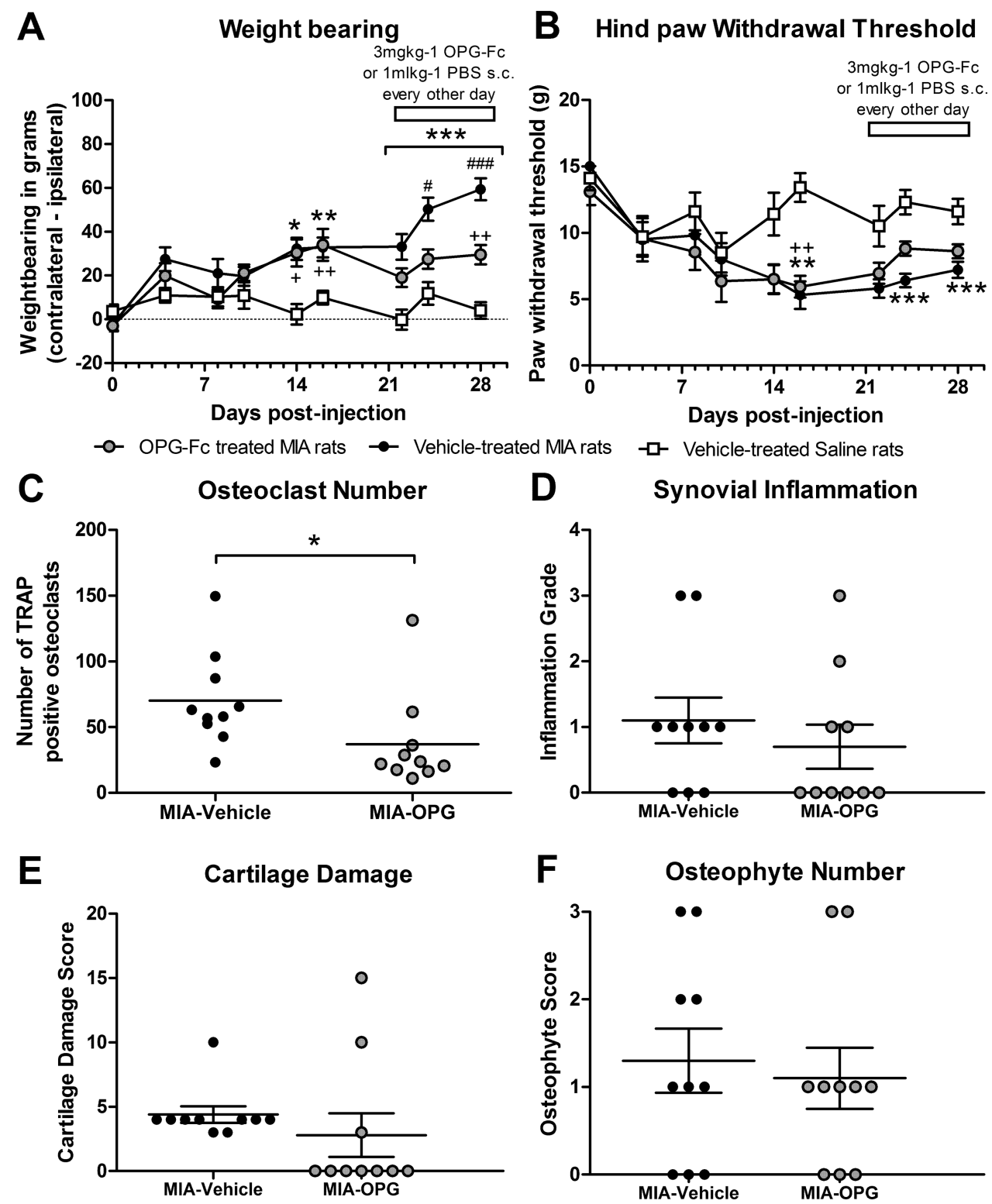

D Synovial Inflammation
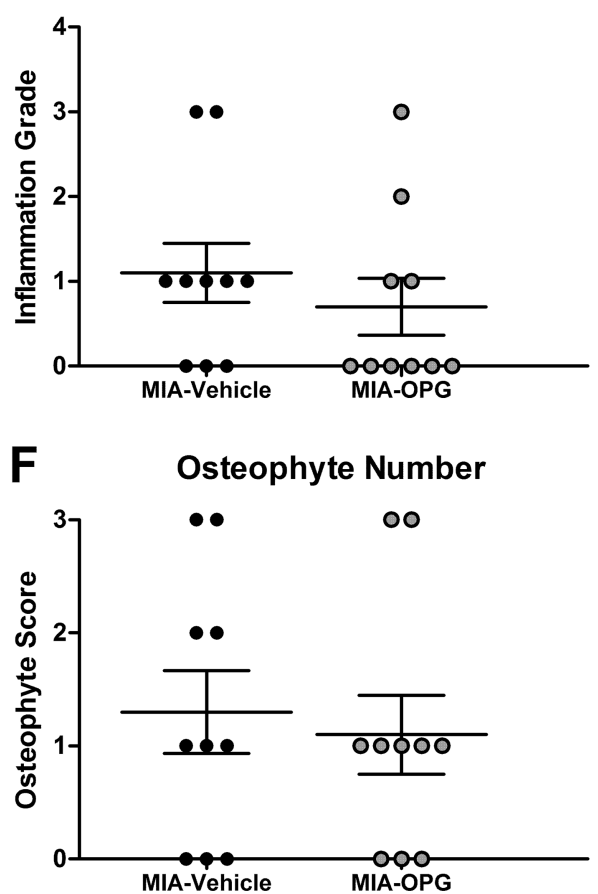

Figure 4 Therapeutic treatment with osteoprotegerin (OPG)-Fc significantly attenuates the further development of changes in (A) weightbearing, but not (B) mechanical allodynia in rats with established monosodium iodoacetate (MIA)-induced joint pathology, which was accompanied by a reduction in (C) osteoclast number. There was no effect on (D) synovial inflammation (E) cartilage damage and (F) osteophyte number. (A and B) Statistical comparison between groups was carried out using a two-way analysis of variance (A) or Kruskal-Wallis test $(B){ }^{*} p<0.05,{ }^{* *} p<0.01$, ${ }^{* * *} p<0.001$ for MIA-vehicle versus saline-vehicle, ${ }^{+} p<0.05,{ }^{++} p<0.01$ for MIA-OPG versus saline-vehicle, ${ }^{\#} p<0.05,{ }^{\# \# \#} p<0.001$ for MIA-OPG versus MIA-vehicle. (C-F) Statistical comparison comparing groups was performed using a Mann-Whitney test. All data are presented as mean $\pm S E M, n=10$ rats per group. For osteoclast numbers six sections for each rat were quantified and data are presented as mean \pm SEM.

and chondrocyte appearance. Therefore preventing these pathological features may not be necessary to relieve pain, at least in the short term.

The reduction in the numbers of osteochondral channels by zoledronate treatment in this study was similar to previous reports, ${ }^{20}$ although the lack of effect of OPG-Fc treatment on osteochondral channel number suggests that maintaining osteochondral integrity is not a primary mechanism underlying the analgesic effects of OPG-Fc in the MIA model of OA pain. Osteophyte scores used in our study were based on size, including cartilaginous surface, and further research would be needed to investigate effects of OPG-Fc on osteophyte ossification. Our findings provide a foundation for future more detailed histomorphometric $^{42}$ or micro-CT studies that would explore other aspects of articular and subchondral bone structure that may mediate pain but could not be measured in this study. It seems likely that the effects of OPG-Fc and zoledronate on pain behaviour, cartilage damage and synovial inflammation may not be attributable to a single mechanism within the subchondral bone.

In conclusion, our data suggest that the early targeting of osteoclast turnover may reduce OA pain as demonstrated in the MIA model of OA pain. Single doses of OPG-Fc and bisphosphonates, based on the literature, have been explored in this study, and other modulators of osteoclasts may also have analgesic potential. ${ }^{16}$ Although OPG is upregulated in human $\mathrm{OA},{ }^{11}{ }^{43}$ this does not appear sufficient to prevent the increased osteoclastogenesis seen clinically. Our data in a model of OA pain suggest that mechanisms contributing to subchondral bone turnover may be particularly important during the early stages 
of OA, and that clinical trials of treatments targeting osteoclast mechanisms are more likely to be successful in early, rather than late, stages of the disease.

Correction notice This article has been corrected since it was published Online First. The first sentence in the Methods section of the abstract has been corrected.

Acknowledgements OPG-Fc was a kind gift from Amgen Ltd.

Contributors DRS, VC, XL, SA, JJB and DAW were involved in the conception and design of the study. All authors were involved in the analysis and interpretation of data, drafting of the article and revising it critically for important intellectual content and final approval of the version to be published.

Funding This work was supported by Arthritis Research UK, grant number 18769.

\section{Competing interests None.}

Provenance and peer review Not commissioned; externally peer reviewed.

Open Access This is an Open Access article distributed in accordance with the Creative Commons Attribution Non Commercial (CC BY-NC 3.0) license, which permits others to distribute, remix, adapt, build upon this work non-commercially, and license their derivative works on different terms, provided the original work is properly cited and the use is non-commercial. See: http://creativecommons.org/ licenses/by-nc/3.0/

\section{REFERENCES}

1 Thorstensson CA, Andersson ML, Jonsson $\mathrm{H}$, et al. Natural course of knee osteoarthritis in middle-aged subjects with knee pain: 12-year follow-up using clinical and radiographic criteria. Ann Rheum Dis 2009;68:1890-3.

2 Graven-Nielsen T, Wodehouse T, Langford RM, et al. Normalization of widespread hyperesthesia and facilitated spatial summation of deep-tissue pain in knee osteoarthritis patients after knee replacement. Arthritis Rheum 2012;64:2907-16.

3 Skou ST, Roos EM, Laursen MB, et al. Total knee replacement plus physical and medical therapy or treatment with physical and medical therapy alone: a randomised controlled trial in patients with knee osteoarthritis (the MEDIC-study). BMC Musculoskelet Disord 2012;13:67.

4 Arendt-Nielsen L, Nie H, Laursen MB, et al. Sensitization in patients with painful knee osteoarthritis. Pain 2010;149:573-81.

5 Suokas AK, Walsh DA, McWilliams DF, et al. Quantitative sensory testing in painful osteoarthritis: a systematic review and meta-analysis. Osteoarthritis Cartilage 2012;20:1075-85.

6 Yusuf E, Kortekaas MC, Watt I, et al. Do knee abnormalities visualised on MRI explain knee pain in knee osteoarthritis? A systematic review. Ann Rheum Dis 2011;70:60-7.

7 Sellam J, Berenbaum F. The role of synovitis in pathophysiology and clinical symptoms of osteoarthritis. Nat Rev Rheumatol 2010;6:625-35.

8 Burr DB, Gallant MA. Bone remodelling in osteoarthritis. Nat Rev Rheumatol 2012;8:665-73.

9 Kwan Tat S, Lajeunesse D, Pelletier JP, et al. Targeting subchondral bone for treating osteoarthritis: what is the evidence? Best Pract Res Clin Rheumatol 2010;24:51-70.

10 Ogino S, Sasho T, Nakagawa K, et al. Detection of pain-related molecules in the subchondral bone of osteoarthritic knees. Clin Rheumatol 2009:28:1395-402.

11 Pantsulaia I, Kalichman L, Kobyliansky E. Association between radiographic hand osteoarthritis and RANKL, OPG and inflammatory markers. Osteoarthritis Cartilage 2010;18:1448-53.

12 Upton AR, Holding CA, Dharmapatni AA, et al. The expression of RANKL and OPG in the various grades of osteoarthritic cartilage. Rheumatol Int 2012;32:535-40.

13 Jimenez-Andrade JM, Mantyh WG, Bloom AP, et al. Bone cancer pain. Ann N Y Acad Sci 2010;1198:173-81.

14 Mapp PI, Walsh DA. Mechanisms and targets of angiogenesis and nerve growth in osteoarthritis. Nat Rev Rheumatol 2012;8:390-8.

15 Laslett LL, Dore DA, Quinn SJ, et al. Zoledronic acid reduces knee pain and bone marrow lesions over 1 year: a randomised controlled trial. Ann Rheum Dis 2012;71:1322-8.

16 Reginster J-Y, Badurski J, Bellamy N, et al. Efficacy and safety of strontium ranelate in the treatment of knee osteoarthritis: results of a double-blind, randomised placebo-controlled trial. Ann Rheum Dis 2013;72:179-86.

17 Hayami T, Pickarski M, Wesolowski GA, et al. The role of subchondral bone remodeling in osteoarthritis: reduction of cartilage degeneration and prevention of osteophyte formation by alendronate in the rat anterior cruciate ligament transection model. Arthritis Rheum 2004;50:1193-206.

18 Bar-Yehuda S, Rath-Wolfson L, Del Valle L, et al. Induction of an antiinflammatory effect and prevention of cartilage damage in rat knee osteoarthritis by CF101 treatment. Arthritis Rheum 2009:60:3061-71.
19 Guzman RE, Evans MG, Bove S, et al. Mono-iodoacetate-induced histologic changes in subchondral bone and articular cartilage of rat femorotibial joints: an animal model of osteoarthritis. Toxicol Pathol 2003:31:619-24.

20 Strassle BW, Mark L, Leventhal L, et al. Inhibition of osteoclasts prevents cartilage loss and pain in a rat model of degenerative joint disease. Osteoarthritis Cartilage 2010;18:1319-28.

21 Moreau M, Rialland P, Pelletier JP, et al. Tiludronate treatment improves structura changes and symptoms of osteoarthritis in the canine anterior cruciate ligament model. Arthritis Res Ther 2011;13:R98.

22 Bingham CO III, Buckland-Wright JC, Garnero P, et al. Risedronate decreases biochemical markers of cartilage degradation but does not decrease symptoms or slow radiographic progression in patients with medial compartment osteoarthritis of the knee: results of the two-year multinational knee osteoarthritis structural arthritis study. Arthritis Rheum 2006:54:3494-507.

23 Pilichou A, Papassotiriou I, Michalakakou K, et al. High levels of synovial fluid osteoprotegerin (OPG) and increased serum ratio of receptor activator of nuclear factor-kappa B ligand (RANKL) to OPG correlate with disease severity in patients with primary knee osteoarthritis. Clin Biochem 2008;41:746-9.

24 Stolina M, Schett G, Dwyer D, et al. RANKL inhibition by osteoprotegerin prevents bone loss without affecting local or systemic inflammation parameters in two rat arthritis models: comparison with anti-TNFalpha or anti-IL-1 therapies. Arthritis Res Ther 2009; 11:R187.

25 Honore $\mathrm{P}$, Luger NM, Sabino MA, et al. Osteoprotegerin blocks bone cancer-induced skeletal destruction, skeletal pain and pain-related neurochemical reorganization of the spinal cord. Nat Med 2000;6:521-8.

26 Kalbhen DA. Chemical model of osteoarthritis-a pharmacological evaluation. J Rheumatol 1987;14(Spec No):130-1.

27 Sagar DR, Staniaszek LE, Okine BN, et al. Tonic modulation of spinal hyperexcitability by the endocannabinoid receptor system in a rat model of osteoarthritis pain. Arthritis Rheum 2010;62:3666-76.

28 Sagar DR, Burston JJ, Hathway GJ, et al. The contribution of spinal glial cells to chronic pain behaviour in the monosodium iodoacetate model of osteoarthritic pain. Mol Pain 2011;7:88

29 Sagar DR, Jhaveri MD, Richardson D, et al. Endocannabinoid regulation of spinal nociceptive processing in a model of neuropathic pain. Eur J Neurosci 2010:31:1414-22.

30 Ashraf S, Mapp PI, Walsh DA. Contributions of angiogenesis to inflammation, joint damage, and pain in a rat model of osteoarthritis. Arthritis Rheum 2011;63:2700-10.

31 Janusz MJ, Hookfin EB, Heitmeyer SA, et al. Moderation of iodoacetate-induced experimental osteoarthritis in rats by matrix metalloproteinase inhibitors. Osteoarthritis Cartilage 2001;9:751-60.

32 Mankin HJ, Dorfman H, Lippiello L, et al. Biochemical and metabolic abnormalities in articular cartilage from osteo-arthritic human hips. II. Correlation of morphology with biochemical and metabolic data. J Bone Joint Surg Am 1971;53:523-37.

33 Mapp PI, Avery PS, McWilliams DF, et al. Angiogenesis in two animal models of osteoarthritis. Osteoarthritis Cartilage 2008;16:61-9.

34 Parfitt AM, Drezner MK, Glorieux FH, et al. Bone histomorphometry: standardization of nomenclature, symbols, and units. Report of the ASBMR Histomorphometry Nomenclature Committee. J Bone Miner Res 1987:2:595-610.

35 Hathway GJ, Vega-Avelaira D, Moss A, et al. Brief, low frequency stimulation of rat peripheral C-fibres evokes prolonged microglial-induced central sensitization in adults but not in neonates. Pain 2009:144:110-18.

36 Pekny M, Pekna M. Astrocyte intermediate filaments in CNS pathologies and regeneration. J Pathol 2004;204:428-37.

37 Nagae $M$, Hiraga $T$, Wakabayashi $H$, et al. Osteoclasts play a part in pain due to the inflammation adjacent to bone. Bone 2006:39:1107-15.

38 Fahlgren A, Messner K, Aspenberg P. Meniscectomy in the rabbit knee leads to increased bone remodelling and cartilage degeneration within three weeks. LiU Electronic Press, 2012. http://liu.diva-portal.org/smash/record.jsf? searchld=3\&pid=diva2:559783 (accessed 10/12/2012).

39 Pickarski M, Hayami T, Zhuo Y, et al. Molecular changes in articular cartilage and subchondral bone in the rat anterior cruciate ligament transection and meniscectomized models of osteoarthritis. BMC Musculoskelet Disord 2011;12:197.

40 Zhang LZ, Zheng HA, Jiang Y, et al. Mechanical and biologic link between cartilage and subchondral bone in osteoarthritis. Arthritis Care Res (Hoboken) 2012;64:960-7.

41 Suri S, Gill SE, Massena de Camin S, et al. Neurovascular invasion at the osteochondral junction and in osteophytes in osteoarthritis. Ann Rheum Dis 2007;66:1423-8

42 Gerwin N, Bendele AM, Glasson S, et al. The OARSI histopathology initiativerecommendations for histological assessments of osteoarthritis in the rat. Osteoarthritis Cartilage 2010;18(Suppl 3):S24-34.

43 Moreno-Rubio J, Herrero-Beaumont G, Tardio L, et al. Nonsteroidal antiinflammatory drugs and prostaglandin $\mathrm{E}(2)$ modulate the synthesis of osteoprotegerin and RANKL in the cartilage of patients with severe knee osteoarthritis. Arthritis Rheum 2010:62:478-88. 